\title{
Design of a liver cancer-specific selector for the analysis of circulating tumor DNA
}

\author{
YAN SUN ${ }^{1-3^{*}}$, RUI MENG ${ }^{4 *}$, HENG TANG $^{5}$, HUIMIN WANG $^{6}$, XUEQIN GUO $^{3}$, YUANYUAN MA $^{2}$, YUN YANG $^{3}$,

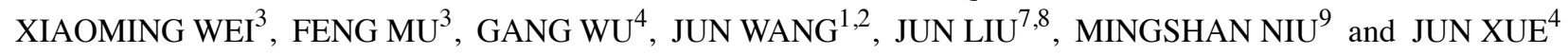 \\ ${ }^{1}$ Department of Biology, University of Copenhagen, Copenhagen DK-2200, Denmark; ${ }^{2}$ BGI Genomics, BGI-Shenzhen, \\ Shenzhen, Guangdong 518083; ${ }^{3}$ Wuhan Medical Laboratory, BGI-Wuhan, Wuhan, Hubei 430075; ${ }^{4}$ Cancer Center, Union \\ Hospital, Tongji Medical College, Huazhong University of Science and Technology, Wuhan, Hubei 430022; ${ }^{5}$ Hefei Anweikang \\ Medical Laboratory, Hefei, Anhui 230000; ${ }^{6}$ Department of Neurology, Xinxiang Central Hospital, Xinxiang, Henan 453000; \\ ${ }^{7}$ Department of Bioscience and Bioengineering, South China University, Guangzhou, Guangdong 510641; \\ ${ }^{8}$ Tianjin Medical Laboratory, BGI-Tianjin, Tianjin 300308; ${ }^{9}$ Jiangsu Key Laboratory of Bone Marrow Stem Cell, \\ Xuzhou Medical College, Xuzhou, Jiangsu 221002, P.R. China
}

Received May 21, 2016; Accepted February 25, 2019

DOI: $10.3892 / \mathrm{ol} .2019 .10243$

\begin{abstract}
Circulating tumor DNA (ctDNA) has been frequently investigated to monitor tumor dynamics and measure tumor burden. This non-invasive method concerning ctDNA has been recognized as a promising biomarker. Recently, next generation sequencing has been used in ctDNA detection by researchers. However, those reports have been limited by modest sensitivity, and only a minority of patients with cancer were applicable. Additionally, a limited number of cases of liver cancer have been analyzed. A more precise method is required to be established to evaluate ctDNA noninvasively. In the present study, a novel method to design a liver cancer-associated chip region (spanning $211 \mathrm{~kb}$, containing 159 genes) was performed with high specificity using International Cancer Genome Consortium datasets. Following evaluation with datasets from The Cancer Genome Atlas and data from 3 patients with liver cancer, the selected regions were demonstrated to be beneficial to locate specific somatic mutations associated with liver cancer therapy and to monitor cancer dynamics in the plasma samples of the patients. In addition to establishing performance benchmarks supporting direct clinical use, the
\end{abstract}

Correspondence to: Mr. Mingshan Niu, Jiangsu Key Laboratory of Bone Marrow Stem Cell, Xuzhou Medical College, 84 West Huaihai Road, Xuzhou, Jiangsu 221002, P.R. China

E-mail:msniu24@126.com

Dr Jun Xue, Cancer Center, Union Hospital, Tongji Medical College, Huazhong University of Science and Technology, 109 Machang Road, Wuhan, Hubei 430022, P.R. China

E-mail: xjunion@126.com

${ }^{*}$ Contributed equally

Key words: selector design, circulating tumor DNA, liver cancer chip designed and the high-resolution sequencing analyses pipeline would allow the development a set of patient specific markers that could monitor the process of cancer with high accuracy and low cost. Furthermore, the present study is essential to understanding the dynamics and providing insight into the basic mechanisms of liver cancer.

\section{Introduction}

Circulating tumor DNA (ctDNA), determined in the cell-free fraction of blood, represents a variable and generally small fraction of the total circulating DNA (1). Plasma-derived ctDNA, determined in the cell-free fraction of blood in patients with cancer, has been recognized as a potential non-invasive biomarker for tumor tissue biopsies (2-4). Next generation sequencing (NGS) studies on ctDNA have revealed that ctDNA is a potential marker associated with various human cancer types (2-4). In 2010, to enhance the clinical management of patients with cancer, Leary et al (2) introduced the concept of using ctDNA for the development of personalized biomarkers to provide an exquisitely sensitive and broadly applicable approach. Van der Vaart et al (3) used a parallel tagged sequencing method to sequence circulating DNA obtained from healthy controls as well as patients with cancer (12 patients with prostate cancer). Chan et al (5) reported the use of shotgun massive parallel sequencing to obtain a non-invasive, genome-wide view of somatic copy number alterations and cancer-associated mutations in four patients with hepatocellular carcinoma (HCC) and a patient with synchronous breast and ovarian cancer, demonstrating the use of ctDNA as a powerful tool for cancer detection, and its potential role as a powerful tool for elucidating important tumoral characteristics, cancer monitoring and research. In a study containing 30 females with metastatic breast cancer who were receiving systemic therapy, Dawson et al (4) compared the radiographic imaging of tumors with the assay of CA 15-3, circulating tumor cells and ctDNA. The results demonstrated 
that ctDNA is an inherently specific, informative and highly sensitive biomarker of metastatic breast cancer. The development of ctDNA is rapid, which indicates notable potentialities and feasibility of using it to monitor tumor dynamics in various solid cancer types. However, the majority of the methods used to test ctDNA are expensive $(3,4)$.

Liver cancer is one of the most common cancer types and cause of cancer-associated mortalities in China (6). Targeted therapies have been achieved by addressing the specific molecular drivers of a patient, which is safer and more efficacious $(1,7,8)$. However, ctDNA with specific tumor mutations has not been extensively investigated or analyzed in patients with liver cancer. The majority of the research used whole genome sequencing to investigate ctDNA (2-5). It has been reported that there are $>6,000$ genes associated with liver cancer, but the majority of which occurred rarely (liverome. kobic.re.kr/index.php). As for the specific nature of ctDNA, sequencing of the sample in depth is required to retrieve the genetic information. Therefore, if the region is too large, it would be a waste of resources, because only the regions associated with liver cancer are required. Furthermore, the majority of the liver cancer-associated genes are not suitable to be markers as the majority of these genes are rarely observed in patients with liver cancer (liverome.kobic.re.kr/index.php). Therefore, these regions specific to liver cancer should be determined. In the present study, liver cancer samples from several databases were used to develop a set of regions specific to liver cancer. Those selected regions were then determined to be suitable for ctDNA analysis. Due to the small size of the designed regions, samples could be sequenced deeply, which would help to make ctDNA a potential marker for cancer monitoring. To the best of our knowledge, this is the first research aiming to develop and design the liver cancer regions for the analysis of ctDNA. Furthermore, the present study could provide valuable information for other cancer chip design types.

In conclusion, in order to detect somatic mutations and design a specific method to quantify ctDNA in liver tumors, a selected region covering multiple classes of somatic mutations that may be identified in patients with liver tumors was designed, and its performance was evaluated in 3 patients with liver cancer. The results provided a set of personalized cancer-specific markers, and evaluated the prognosis of therapy.

\section{Materials and methods}

Data analysis pipeline. In the present study, a data analysis pipeline, including data filtration, alignment, variants detection and results annotation for whole genome data and data in the selected regions, was established (Fig. 1). The sequencing data (bam file) were provided by Professor Yuk-Ming Dennis Lo from The Chinese University of Hong Kong (Hong Kong, China). The bam file was sorted, and bedtools (bedtools version 2.25.0) was used to change the bam file into an fq file, via filtering of certain reads with the same read ID, for further analysis. The pipeline started from the clean reads, as follows: Firstly, the clean reads with a length of $50 \mathrm{bps}$ were mapped to the human reference genome (hg19) from the University of California, Santa Cruz database (hgdownload.soe.ucsc.edu/goldenPath/hg19/bigZips/) using BWA (Burrows Wheeler Aligner; bio-bwa.sourceforge. net/); secondly, following removal of polymerase chain reaction-derived duplications using Picard (broadinstitute.github. io/picard/) and realigning by GATK (https://software.broadinstitute.org/gatk/documentation/tooldocs/current/), the bam results were then used to determine variant detection. Somatic SNVs calling were performed using MuTect (software.broadinstitute. org/cancer/cga/mutect). To reduce false-positives, stringent criteria were used in the present study, as follows: Firstly, a mutation was kept only when it was completely absent in the result blood sample; secondly, a mutation was kept only when the sequencing depth was $>20$-fold. This threshold was applied to lower the false-positive detection rate. Somatic Indel calling was performed using Varscan software (varscan.sourceforge. net/). Detailed filtering parameters followed the best practice guidelines (varscan.sourceforge.net/using-varscan.html). Local realignment around Indels was also included. The identified somatic variants were directly annotated by the Catalogue of Somatic Mutations in Cancer (COSMIC, https://cancer.sanger. ac.uk/cosmic/) database, dbSNP, Hapmap, 1000 Genome and dbNSFP using our own PERL scripts if the alterations have been reported to be disease-causing mutations or targets for therapy.

Design of a liver cancer-associated selector. In the present study, the focus was on liver cancer; therefore, specific regions, including the coding sequences (CDSs), covering recurrent alterations in potential recurrent mutated genes were designed. Using the method by Newman et al (9) a novel algorithm to determine the liver cancer-associated regions was developed. Firstly, a list of genes covering recurrent alterations in potential driver genes were selected using the top 100 genes listed in COSMIC (10). Subsequently, regions of CDS containing recurrent single nucleotide variants (SNVs) were selected based on the mutation frequency of the CDS region (cut-off value, 5; Fig. 2) to form potential liver cancer-associated regions. Following this, to maximize the number of mutations per patient while minimizing the region, a novel algorithm was applied using whole-exome sequencing (WES) data from 243 patients (the LINC-JP_Liver_Cancer-NCC_JP project by December 6, 2014) with liver cancer with cancer-associated SNVs and Indels profiled by International Cancer Genome Consortium (https://icgc.org/). Of the 243 patients included in the study, $74.49 \%$ (181/243) were male, and the age of the 243 patients at diagnosis ranged from 23-85 years. Detailed information of the patients can be found in the ICGC database (https://icgc.org/icgc/cgp/66/420/824). Additionally, a number of genomic regions harboring liver cancer driver genes known to be associated with liver cancer therapy were included. The present study and the protocols used were approved by the Institutional Ethics Committee of BGI (Shenzhen, China).

The main steps and cut off parameters are as follows (Fig. 2A): i) Step 1: Region 1. To select the CDS region (initial region 1) covering recurrent alterations in known driver genes, the data of patients with liver cancer from COSMIC was analyzed. Subsequently 'mutation frequency' in a specific gene region in patients with liver cancer was used to select the initial seed genes. The cut-off value was 12 (Fig. 2B), and 100 genes from the COSMIC patients with liver cancer were obtained. Subsequently, 'mutation frequency' of the CDS region (Fig. 2C; cut-off value was 5) was used to select the initial seed regions and a region with 141 CDS regions was 


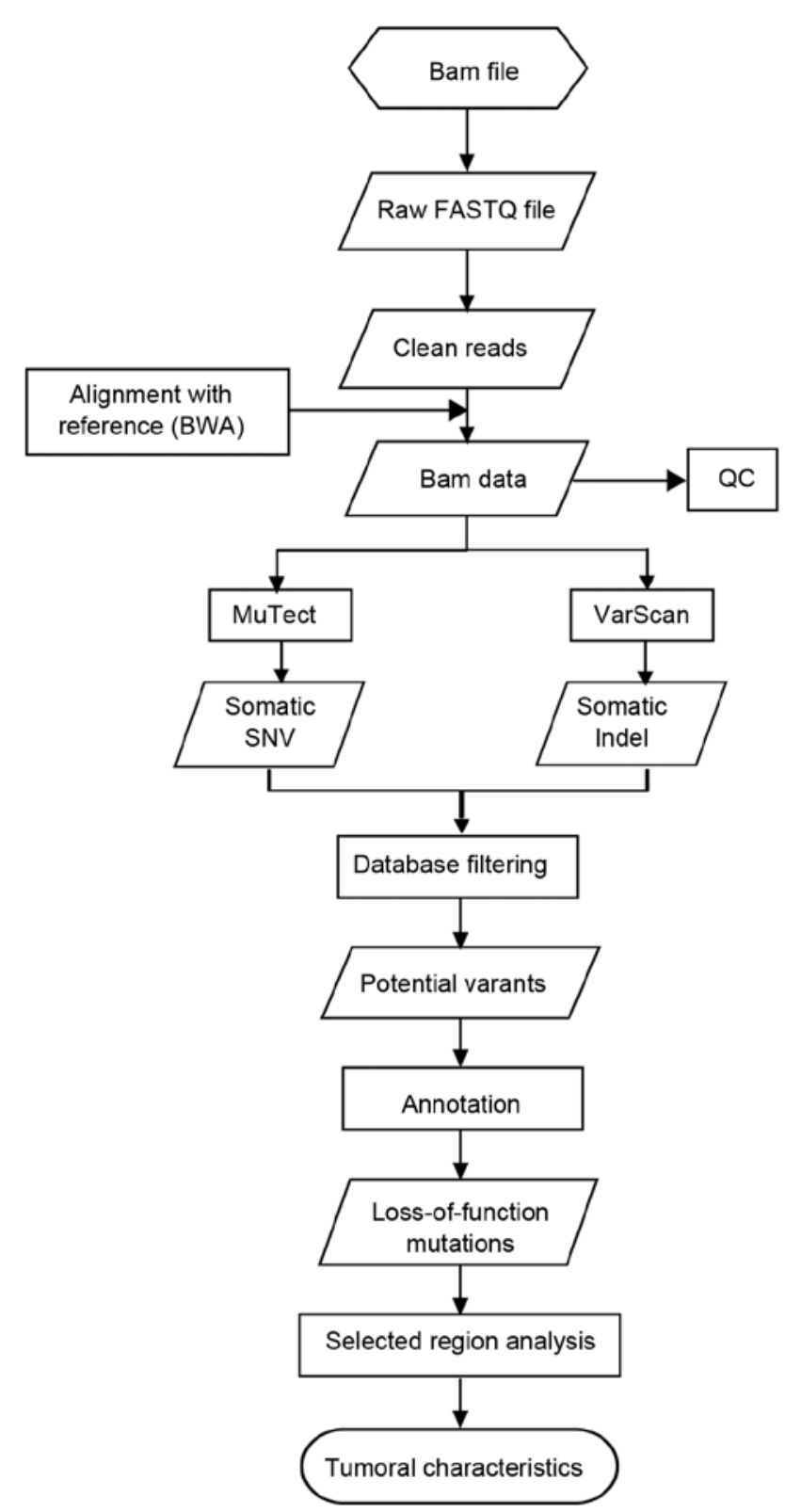

Figure 1. Bioinformatics pipeline. Flow diagram of bioinformatics analysis. QC, quality control.

obtained. The final region 1 was generated from the intersection of the seed genes and the CDS regions. Finally, region 1 with 122 CDS regions was selected (data not shown);

ii) Step 2: Region 2. To maximize the number of mutations per patient while minimizing the region, a novel algorithm was used using WES data from 243 patients (the LINC-JP_Liver Cancer-NCC_JP project) with cancer-associated SNVs and Indels profiled by International Cancer Genome Consortium (icgc.org/). Firstly, patients with a mutation in region 1 were removed. A total of 142 samples were removed, which indicated a high liver cancer-associated nature of region 1. The remaining samples were retained for further analysis. Secondly, the 'sample frequency' of all the CDSs in the remaining patients was calculated. A cut off value of 4 was used in this step (Fig. 2D). Thirdly, the remaining CDS region, which would be selected only if at least one new patient was identified, was analyzed. This was repeated until no further region met these criteria. In this step, region 2 (69 CDS regions) was selected. Additionally, 91 more samples were covered in this step, and a total of 233 samples (233/243) were covered in regions 1 and 2 .

iii) Step 3: Region 3. In this step, 'sample frequency' and the recurrence index (RI) value were used. Firstly, a sample cutoff value of 2 and RI cutoff value of 10 was used to generate a seed list of 257 candidate CDS regions. The cutoff value aforementioned is not a stringent filtering parameter. Therefore, the 'mutation frequency' of the aforementioned seed CDS regions was calculated. A cutoff value of 3 was used, which means that only the CDS regions with at least 3 mutations were obtained. Region 3 was obtained in this step, which contains 14 CDS regions in total (region 3).

iv) Step 4: Add chemotherapy-associated site. Single nucleotide polymorphisms, which were reported to be associated with chemotherapy (region 4) were included.

v) Step 5: Add targeted drug-associated sites. By the time of submission of this paper, there has only been one drug demonstrated to treat liver cancer, Sorafenib, which blocks the $\mathrm{RAF} /$ mitogen-activated protein kinase kinase/extracellular signal-regulated kinase pathway (11). A number of potential targeted genes reported to be targetable to some drugs (region 5) were also included.

Selected regions performance assessment. To investigate the specificity of the selected genomic regions selected, WES data from patients with liver cancer-associated SNVs and Indels profiled by TCGA (by January 10, 2015) were used for assessment. The aim was to evaluate the fraction of the 192 patients containing at least one loss-of-function mutation in the selected regions.

Evaluation of somatic mutation detection in 3 patients with liver cancer. To investigate the performance of the designed regions, 3 patients with liver cancer were recruited, who all received surgery [formalin-fixed paraffin embedded (FFPE) sample and blood sample sequencing data prior to surgery, and ctDNA sequencing data prior to and following surgery]. The samples were collected in the Prince of Wales Hospital (Hong Kong, China), as previously described (5). Written informed consent was obtained from all the participants at the time of sample collection. The sequencing data was provided by Professor Yuk-Ming Dennis Lo from The Chinese University of Hong Kong. The following pipeline was used to identify patient specific mutations, and then evaluate the performance of the designed regions in monitoring cancer in the 3 patients. The bioinformatics pipeline is depicted in Fig. 1.

Statistical analysis. To assess statistical significance, random selectors were selected using the same size to the present selected region. The performance of random selectors and liver cancer-associated selectors were compared (Z-test), and P-values were calculated accordingly.

\section{Results}

Liver cancer-associated selector. The following algorithm was used to design the liver cancer-associated selector, aiming to collect all the liver cancer-associated genes and regions. 
A

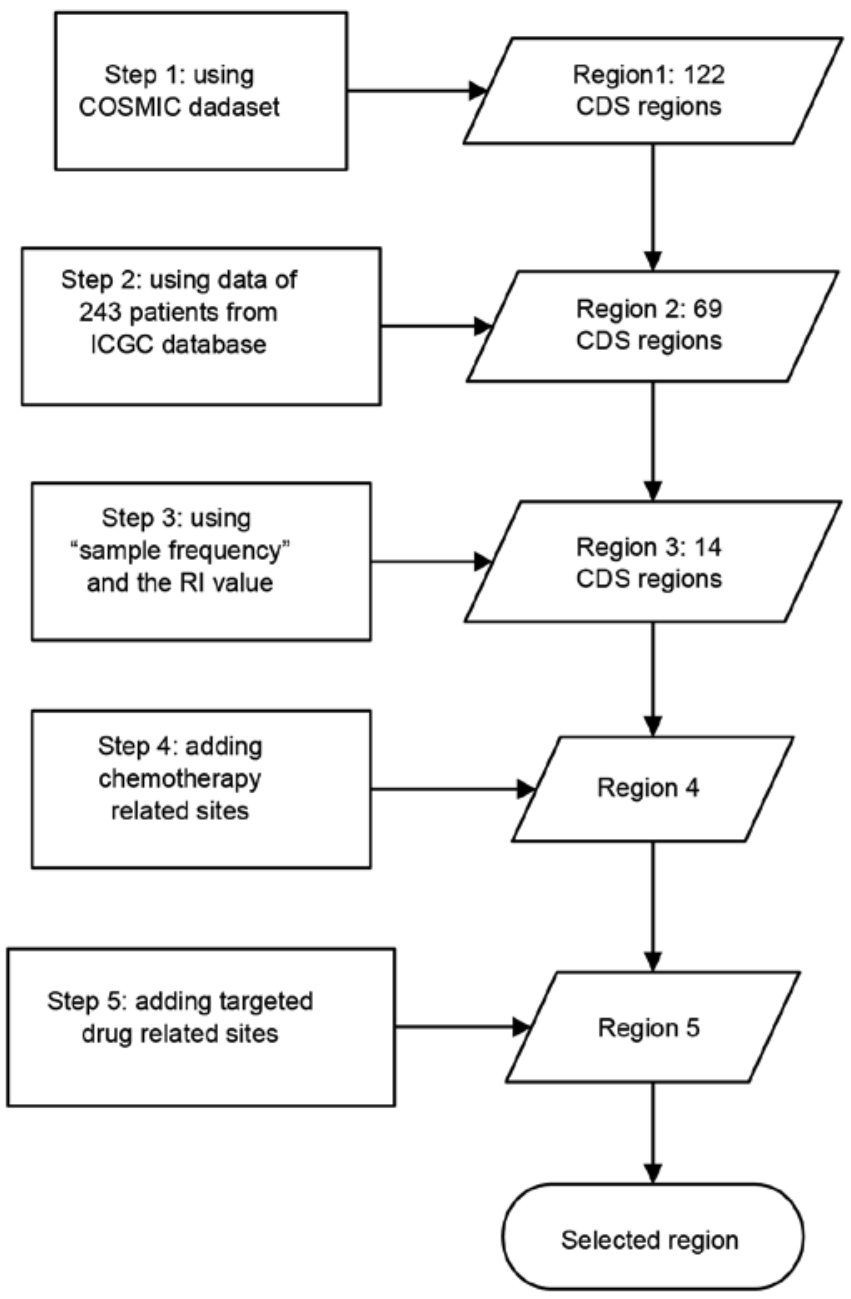

B

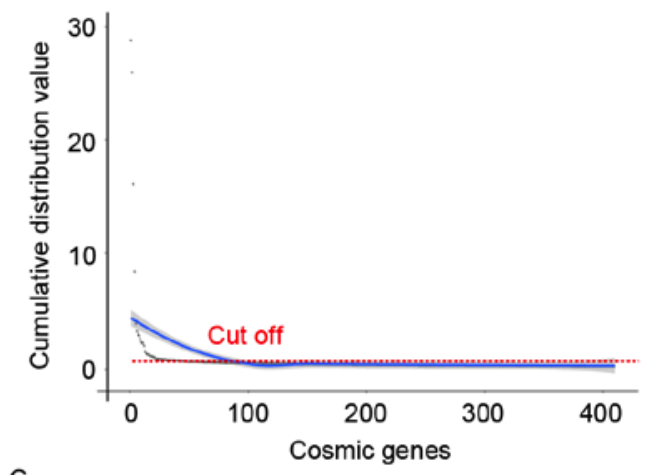

C

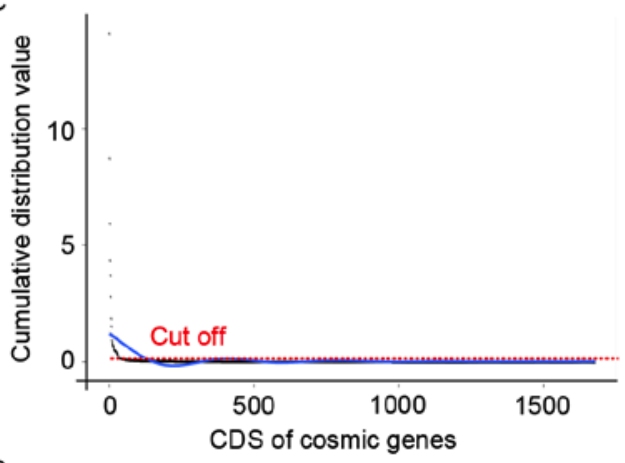

D

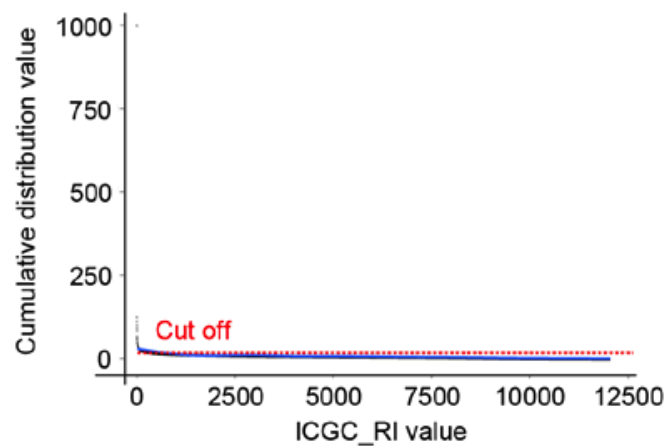

Figure 2. Selected region design workflow. (A) Selected region design workflow. (B) Cut-off value used to selected the initial seed genes. (C) Cut-off value used to select the initial seed region 1. (D) Cut-off 'sample frequency' of all the CDSs using data from ICGC. ICGC, International Cancer Genome Consortium; COSMIC, Catalogue of Somatic Mutations in Cancer. RI, recurrence index; CDS, coding sequence.

In the whole process, two values were primarily introduced to maximize the association of the selected regions and liver cancer, whilst minimizing the length of the designed regions: One was 'mutation frequency', which is defined as the number of SNVs/Indels that occur within a given cohort of patients; the other one was 'sample frequency', which is defined as the number of the samples (carrying specific mutations) in a specific CDS region. The number of patients with mutations/CDS length in kb [known as RI (9)] was used to measure patient-level recurrence frequency at the CDS level. This RI could normalize gene or CDS size.

Following all of the aforementioned steps, a specific region of $211 \mathrm{~kb}$, which included 159 genes, was obtained.

Reliability and accuracy evaluation of the selector. To investigate the specificity of the aforementioned selected genomic regions, WES data from 192 patients with liver cancer-associated SNVs and Indels profiled by TCGA were used for assessment. Detailed information of the patients can be found in TCGA database (by January 10, 2015). A total of 107 patients $(107 / 192,55 \%)$ contained at least one loss-of-function mutation in the selected region. This independent cohort contained a mean of 284.4 mutations following the removal of 5 samples, which contained $>100,000$ mutations each and would influence the sample frequency. The RI of the selected region in the remaining samples was 1 , which means the number of patients with loss-of-function mutations/CDS length in $\mathrm{kb}$ was $>1$. To assess the statistical significance, a $211 \mathrm{~kb}$ region was randomly selected for comparison. A mean $\mathrm{RI}$ of 0.0123 was determined in the randomly selected region $\left(\mathrm{P}<1.0 \times 10^{-5}\right.$ for the difference between random selectors and liver cancer-associated selector; Z-test), which validated a high specificity of the selected genomic regions to liver cancer.

Analysis of the 3 patients with liver cancer. To demonstrate the use of the designed region in elucidating notable tumoral characteristics, and the potential role of ctDNA as a powerful biomarker, the WGS (whole genome sequencing) data of 
3 patients with HCC (blood and FFPE pre-resection samples, and pre- and post-resection plasma samples), which were recruited for another study (5), were analyzed. The analysis was started from the bam file provided by Professor Yuk-Ming Dennis Lo from The Chinese University of Hong Kong. The bam file was sorted first, and then bedtools was used to change the bam file into a fq file for further analysis. During this process, some reads with the same read ID were removed. Subsequently, the aforementioned pipeline was used for analysis. The sequencing results are detailed in Table I.

For all the samples included in the present study, a mean of 1,079,369,231 reads were mapped to regions of the hg19 genome, resulting in a 23 -fold average depth (Table I). The mean coverage of the 3 blood samples (all sample types) was $>91.5 \%$, except FFPE sample T013 and T027. The mean sequencing depth of the blood sample, FFPE samples and plasma sample was 31.7, 27.3 and 16.6, respectively, which demonstrated a relatively low sequencing depth of the plasma sample. The data in the selected region was then removed from the WGS data for further analysis. The mean coverage of the 3 patients (all sample types) in the selected region was $~ 98 \%$, except for sample T27, while the mean sequencing depth of the blood sample, FFPE samples and plasma sample of the selected region was $53.5,68.5$ and 20.5 , respectively.

No Indels were identified in the selected regions; therefore, the primary focus was on the somatic SNVs. The total number of detected SNVs was detailed in Table II. There was a mean of 3,097 mutations detected in the whole genome region of all the tissue samples. The mutations determined in the plasma prior to and following surgery are almost equal, which made it difficult to annotate and generate the association between those mutations and the cancer states. While the mutations located in the selected region were relative small and liver-cancer associated. The comparison between whole genome data and data of the selected region was aimed to reveal its potential role as a powerful tool for cancer detection and monitoring. As detailed in Table II, the mean detected mutations of the FFPE samples and plasma sample of the designed selected region was 71.3 and 5.5 , respectively.

Monitoring of serial ctDNA levels of the liver cancerassociated selector. The specific pattern(s) of ctDNA involved in the disease states identified by targeted NGS sequencing may provide a set of biomarker/therapeutic that could monitor tumor dynamics with high accuracy, which may be used as a more precise diagnostic tool to predict disease risk and treatment responses.

The performance of circulating biomarkers from the selected regions in the 3 patients with HCC prior to and following surgery was analyzed (Fig. 3). This was performed to evaluate whether the fluctuations of ctDNA in the selected regions can reflect the dynamics of the disease. The significantly detectable levels of ctDNA data from the selected region may be used to determine tumor volumes and clinical responses to therapy.

In the present study, the analysis of the reads ratio in the selected region was performed to monitor the effect of surgery. The reads ratio was defined as the fraction of mutated reads in all detected reads. The reads ratio in patients 2 and 3 was notably reduced following surgery (Fig. 3). Those two patients exhibited similar dynamic patterns, which may reflect tumor burden in plasma samples. However, further experiments are required to be performed to support this result.

There were $6 \mathrm{SNVs}$ exhibited in the pre-surgery sample of patient 1. A total of three mutations were not identified in the post-surgery sample of patient 1, including: TERT c.C1310G and c.G555C, and MET c.T1621G, which may partially reflect the clinical effect of surgical treatment. However, three mutations (ALK c.A2242G, NOTCH1 c.A4111C and FLCN c.T884G) in the pre-surgery sample of patient 1 were also exhibited in the post-surgery sample. These minimal residuals may indicate possible progression of occult microscopic disease. These data may highlight the promise of ctDNA analysis from the specific selector region for identifying patients with residual disease following treatment.

Somatic mutation reads upregulated/downregulated, according to the treatment sensitivity of the patients, are considered to be novel biomarkers. In the present study, the results in the selected region demonstrated that the present method provided an optimal method, including the design of specific liver cancer-associated selector, and then the monitoring of treatment response, which could provide a more accurate, sensitive and economic method, compared with whole genome sequencing. If the data indicates a strong association between ctDNA level and therapy sensitivity of the patients, it will provide evidence of the role of ctDNA in cancer monitoring, and may further validate previous investigations.

\section{Discussion}

Continuing ineffective therapies and unnecessary side effects are common limitations of cancer treatment (12), and thus far there is no effective method to monitor treatment response, and to determine the benefit of novel therapeutics. Generally, the use of serial imaging, including radiographic measurements, in assessing treatment response and detecting changes in tumor burden, frequently fails. For example, patients with non-small cell lung cancer undergoing definitive radiotherapy frequently have surveillance computed tomography (CT)/positron emission tomography (PET)-CT scans that are difficult to interpret due to fibrotic changes and radiation-induced inflammatory in the lung and surrounding tissues (13). Thus, there is an urgent requirement for more sensitive and specific biomarkers to measure tumor burden. Along with the development of NGS, the research of ctDNA has become a hot spot in this field. It can be used as a potential marker to predict disease risk, patient outcomes or response to treatment (2-4). However, the reports published thus far have a number of limitations. Firstly, it is difficult to detect low frequency mutations; secondly, the acute monitoring of ctDNA requires the detection of somatic mutations in tumor-tissue samples, which are required to be performed in an invasive way; and finally, evaluation of the ctDNA levels is different in reported articles. For example, certain studies used absolute levels of ctDNA or fraction of mutant allele in plasma to represent the ctDNA levels $(4,14)$, while other studies used the mean allele frequency of the major clone to represent the ctDNA levels (15). This inconsistency makes it difficult to use ctDNA as a biomarker to monitor tumor dynamics in patients with various solid types of cancer. 
Table I. Whole genome and selected region performance results.

\begin{tabular}{|c|c|c|c|c|c|c|c|}
\hline Sample & Type & $\begin{array}{l}\text { Mapped } \\
\text { reads }\end{array}$ & $\begin{array}{c}\text { Duplicate } \\
\text { rate }(\%)\end{array}$ & $\begin{array}{c}\text { Whole } \\
\text { genome } \\
\text { coverage }(\%)\end{array}$ & $\begin{array}{l}\text { Coverage } \\
\text { of selected } \\
\text { regions }(\%)\end{array}$ & $\begin{array}{l}\text { WGS } \\
\text { sequencing } \\
\text { depth }\end{array}$ & $\begin{array}{l}\text { Sequencing depth } \\
\text { of selected } \\
\text { regions }\end{array}$ \\
\hline case $013 \mathrm{~W}$ & Blood & $1,004,693,827$ & 1.89 & 92.58 & 98.59 & 25.49 & 42.63 \\
\hline T013 & FFPE & $1,082,465,782$ & 4.09 & 86.08 & 98.04 & 26.86 & 58.93 \\
\hline Plsm013_pre & Plasma & $951,847,727$ & 2.34 & 92.05 & 98.08 & 16.04 & 18.73 \\
\hline Plsm013_post & Plasma & $1,074,184,598$ & 2.02 & 92.20 & 98.48 & 18.16 & 22.03 \\
\hline case $023 \mathrm{~W}$ & Blood & $971,811,353$ & 2.00 & 92.57 & 98.59 & 24.64 & 41.16 \\
\hline T023 & FFPE & $1,128,719,103$ & 2.04 & 92.57 & 98.40 & 28.62 & 49.26 \\
\hline Plsm023_pre & Plasma & $881,754,515$ & 1.77 & 91.52 & 98.24 & 14.99 & 21.35 \\
\hline Plsm023_post & Plasma & $987,003,992$ & 2.42 & 92.02 & 98.38 & 16.66 & 23.36 \\
\hline case $027 \mathrm{~W}$ & Blood & $1,810,697,288$ & 3.97 & 92.83 & 98.58 & 44.98 & 76.61 \\
\hline T027 & FFPE & $1,064,991,038$ & 4.20 & 74.04 & 93.20 & 26.41 & 97.33 \\
\hline Plsm027_pre & Plasma & $928,705,384$ & 2.88 & 92.24 & 97.66 & 15.56 & 16.66 \\
\hline Plsm027_post & Plasma & $1,065,556,168$ & 2.10 & 92.32 & 98.29 & 18.00 & 20.91 \\
\hline
\end{tabular}

FFPE, formalin-fixed paraffin embedded; WGS, whole genome sequencing.

Table II. Analysis results of somatic SNVs.

\begin{tabular}{|c|c|c|c|c|c|}
\hline Patient & Gender & Age, years & Sample pair & Somatic SNVs & Somatic SNVs in selected region \\
\hline \multirow[t]{3}{*}{ Patient 1} & Male & 77 & case013W-T013 & 3,500 & 75 \\
\hline & & & case013W-Plsm013_pre & 274 & 6 \\
\hline & & & case013W-Plsm013_post & 330 & 7 \\
\hline \multirow[t]{3}{*}{ Patient 2} & Male & 58 & case $023 \mathrm{~W}-\mathrm{T} 023$ & 3,060 & 86 \\
\hline & & & case023W-Plsm023_pre & 345 & 10 \\
\hline & & & case023W-Plsm023_post & 306 & 6 \\
\hline \multirow[t]{3}{*}{ Patient 3} & Male & 39 & case027W-T027 & 2,732 & 53 \\
\hline & & & case027W-Plsm027_pre & 98 & 3 \\
\hline & & & case027W-Plsm027_post & 114 & 1 \\
\hline
\end{tabular}

SNVs, single nucleotide variants.

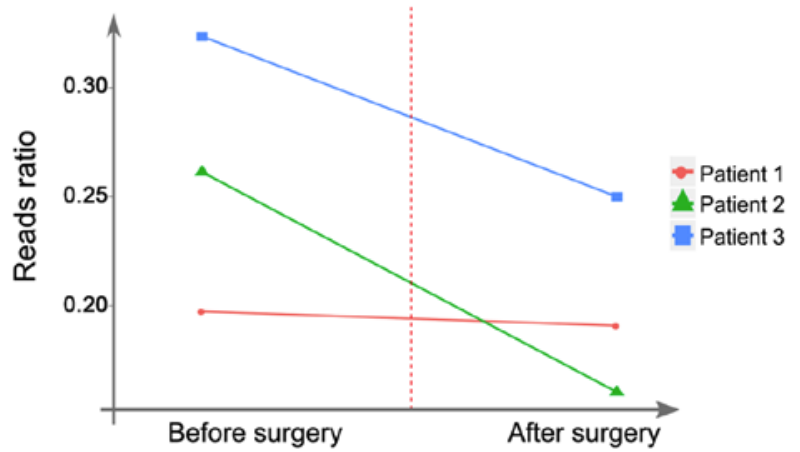

Figure 3. Results of the reads ratio in the selected region to monitor the effect of surgery.

To solve those problems, the aim of the present study was to develop a novel method. Firstly, based on considerations of high sequencing depth, a selector only containing liver-associated genes was designed; and secondly, the aim was to establish a more precise target in analyzing ctDNA in cancer monitoring. In the present study, patient specific mutation sites were analyzed instead of the whole ctDNA level through targeted NGS sampling at different times. This could make ctDNA an inherently specific, informative and highly sensitive biomarker for liver cancer.

There are $>6,000$ genes reported to be liver cancer associated (liverome.kobic.re.kr/index.php). To the best of our knowledge, no available database provides a selector specific for liver cancer. Only a minority of tumor types can be defined using a small number of recurrent mutations at predefined positions. If the region is too large (such as WGS/WES), the cost of sequencing would increase substantially. The cost greatly influences the clinical application of ctDNA detection, meaning that the design of the specific selector is vital in the present study. First, it dictates which mutations can be detected with high probability for a patient with a particular cancer 
type; and secondly, the selector size directly impacts the cost and depth of sequence coverage. For example, the smaller the selector is, the deeper the sequencing depth could be achieved.

In the present study, a novel algorithm was used in a liver cancer-associated selector design. It was considered that 'mutation frequency' and 'sample frequency' should be considered at the same time. In the first step of process of designing a liver cancer-associated selector, genes and CDS were taken into consideration to generate region 1 , due to it being considered that genes are functional elements, which could be used as the first filter, and CDS regions could be used to minimize the regions associated with liver cancer mutations. To determine the regions most associated with liver cancer, the algorithm used was more stringent in the first 3 steps, particularly in the third step, where 3 values were used to calculate and filter.

The mean sequencing depth of the blood sample, FFPE samples and plasma sample of the selected region was 53.5, 68.5 and 20.5 , respectively, which demonstrated a relatively low sequencing depth of the plasma samples. The coverage of FFPE sample T013 and T027 was 86.08 and $74.04 \%$, respectively, which may be a result of degradation of the samples. Only the associations of SNV and disease progression were discussed in the present study. The whole genome CNV status has been investigated in previous research (5). The key point in the present study is the sensitivity of designed selector.

A number of researchers reported that the absolute levels of ctDNA in pretreatment plasma were significantly correlated with tumor volume, as measured by CT and PET imaging (2-4). Therefore, they used total volume of ctDNA as a marker. However, in a number of cases, it has also been observed that certain mutations dominated the plasma $(4,14,16)$. As a result, it was considered that mutations in potential driver genes or actionable mutations could better reflect the tumor dynamics. Analysis of the total reads in the selected region was conducted to monitor the effect of surgery. The reads ratio in patients 2 and 3 is notably reduced following surgery (Fig. 3), which demonstrated similar dynamic patterns in plasma. To support this result, more experiments are required to be performed. However, the ratio of the ctDNA remained detectable in the plasma following surgery, which is the reason why further investigation is required.

Along with the development and improvement of NGS, the research of ctDNA has become a hot spot in this field. The monitoring of ctDNA levels requires the identification of somatic mutations in patients with cancer. However, due to the high cost of sequencing, the characteristic of ctDNA slowed down the application of ctDNA in the clinical setting. The present study provided an optimal method to design a cost-effective alternative for ctDNA analysis, and targeted deep sequencing can be readily expanded to include other genes known to be recurrently mutated in a specific cancer type. Therefore, Chinese HCC samples will be collected for testing in future work.

\section{Acknowledgements}

The authors would like to thank Professor Yuk-Ming Dennis Lo from The Chinese University of Hong Kong (Hong Kong,
China) for providing access to the sequencing data of the patients with hepatocellular carcinoma.

\section{Funding}

The present study was supported by National Natural Science Foundation of China (grant no. 81502593).

\section{Availability of data and materials}

The datasets generated and/or analyzed during the present study are available in the European Genome-Phenome Archive (EGA; www.ebi.ac.uk/ega/), which is hosted by the European Bioinformatics Institute (EBI), under accession number EGAS00001000370.

\section{Authors' contributions}

YS, RM, HT, HW, JX, GW and JW designed the research and wrote the first draft of the article. JL, XG, YM, YY, XW, FM and $\mathrm{MN}$ contributed to revising the manuscript critically. YS, HT, HW and MN developed the algorism. YS, RM, JX, HT, HW, MN, XG, YM, YY, XW, FM and JL performed the data analysis. All authors approved the final version to be published.

\section{Ethics approval and consent to participate}

The present study was approved by the ethics committee of BGI-Shenzhen (Shenzhen, China). Written informed consent was obtained from all the participants at the time of sample collection.

\section{Patient consent for publication}

Not applicable.

\section{Competing interests}

The authors declare that they have no competing interests.

\section{References}

1. Ignatiadis M and Dawson SJ: Circulating tumor cells and circulating tumor DNA for precision medicine: Dream or reality? Ann Oncol 25: 2304-2313, 2014.

2. Leary RJ, Kinde I, Diehl F, Schmidt K, Clouser C, Duncan C, Antipova A, Lee C, McKernan K, De La Vega FM, et al: Development of personalized tumor biomarkers using massively parallel sequencing. Sci Transl Med 2: 20ra14, 2010.

3. van der Vaart M, Semenov DV, Kuligina EV, Richter VA and Pretorius PJ: Characterisation of circulating DNA by parallel tagged sequencing on the 454 platform. Clin Chim Acta 409: 21-27, 2009.

4. Dawson SJ, Tsui DW, Murtaza M, Biggs H, Rueda OM, Chin SF, Dunning MJ, Gale D, Forshew T, Mahler-Araujo B, et al: Analysis of circulating tumor DNA to monitor metastatic breast cancer. N Engl J Med 368: 1199-1209, 2013.

5. Chan KC, Jiang P, Zheng YW, Liao GJ, Sun H, Wong J, Siu SS, Chan WC, Chan SL, Chan AT, et al: Cancer genome scanning in plasma: Detection of tumor-associated copy number aberrations, single-nucleotide variants, and tumoral heterogeneity by massively parallel sequencing. Clin Chem 59: 211-224, 2013.

6. Chen JG and Zhang SW: Liver cancer epidemic in China: Past, present and future. Semin Cancer Biol 21: 59-69, 2011. 
7. Mabert K, Cojoc M, Peitzsch C, Kurth I, Souchelnytskyi S and Dubrovska A: Cancer biomarker discovery: Current status and future perspectives. Int J Radiat Biol 90: 659-677, 2014

8. Pantel $\mathrm{K}$ and Alix-Panabieres C: Real-time liquid biopsy in cancer patients: Fact or fiction? Cancer Res 73: 6384-6388, 2013.

9. Newman AM, Bratman SV, To J, Wynne JF, Eclov NC, Modlin LA, Liu CL, Neal JW, Wakelee HA, Merritt RE, et al: An ultrasensitive method for quantitating circulating tumor DNA with broad patient coverage. Nat Med 20: 548-554, 2014.

10. Forbes SA, Tang G, Bindal N, Bamford S, Dawson E, Cole C, Kok CY, Jia M, Ewing R, Menzies A, et al: COSMIC (the Catalogue of Somatic Mutations in Cancer): A resource to investigate acquired mutations in human cancer. Nucleic Acids Res 38 (Database Issue): D652-D657, 2010.

11. Lang L: FDA approves sorafenib for patients with inoperable liver cancer. Gastroenterology 134: 379, 2008

12. Kumar B, Singh S, Skvortsova I and Kumar V: Promising targets in anti-cancer drug development: Recent updates. Curr Med Chem 24: 4729-4752, 2017.

13. Jahangiri P, Pournazari K, Torigian DA, Werner TJ, Swisher-McClure S, Simone CB II and Alavi A: A prospective study of the feasibility of FDG-PET/CT imaging to quantify radiation-induced lung inflammation in locally advanced non-small cell lung cancer patients receiving proton or photon radiotherapy. Eur J Nucl Med Mol Imaging 46: 206-216, 2019.
14. Forshew T, Murtaza M, Parkinson C, Gale D, Tsui DW, Kaper F, Dawson SJ, Piskorz AM, Jimenez-Linan M, Bentley D, et al: Noninvasive identification and monitoring of cancer mutations by targeted deep sequencing of plasma DNA. Sci Transl Med 4: 136ra168, 2012.

15. Murtaza M, Dawson SJ, Pogrebniak K, Rueda OM, Provenzano E, Grant J, Chin SF, Tsui DW, Marass F, Gale D, et al: Multifocal clonal evolution characterized using circulating tumour DNA in a case of metastatic breast cancer. Nat Commun 6: 8760, 2015.

16. Kidess E, Heirich K, Wiggin M, Vysotskaia V, Visser BC, Marziali A, Wiedenmann B, Norton JA, Lee M, Jeffrey SS and Poultsides GA: Mutation profiling of tumor DNA from plasma and tumor tissue of colorectal cancer patients with a novel, high-sensitivity multiplexed mutation detection platform. Oncotarget 6: 2549-2561, 2015.

(i) () (9) This work is licensed under a Creative Commons Attribution-NonCommercial-NoDerivatives 4.0 International (CC BY-NC-ND 4.0) License. 